\title{
Biochemical Engineering of Cell Surface Sialic Acids Stimulates Axonal Growth
}

\author{
Bettina Büttner, ${ }^{1 *}$ Christoph Kannicht, ${ }^{2 *}$ Carolin Schmidt, ${ }^{1}$ Klemens Löster, ${ }^{2}$ Werner Reutter, ${ }^{1}$ Hye-Youn Lee, \\ Sabine Nöhring, ${ }^{1}$ and Rüdiger Horstkorte ${ }^{1}$ \\ 1'Institut für Molekularbiologie und Biochemie, Fachbereich Humanmedizin, Freie Universität Berlin, D-14195 Berlin- \\ Dahlem, Germany, and 2Octapharma Pharmaceutica, A-1100 Vienna, Austria (Department-Unit for Molecular \\ Biochemistry, D-14195 Berlin-Dahlem, Germany)
}

Sialylation is essential for development and regeneration in mammals. Using $N$-propanoylmannosamine, a novel precursor of sialic acid, we were able to incorporate unnatural sialic acids with a prolonged $\mathrm{N}$-acyl side chain (e.g., $\mathrm{N}$-propanoylneuraminic acid) into cell surface glycoconjugates. Here we report that this biochemical engineering of sialic acid leads to a stimulation of neuronal cells. Both PC12 cells and cerebellar neurons showed a significant increase in neurite outgrowth after treatment with this novel sialic acid precursor. Furthermore, also the reestablishment of the perforant pathway was stimulated in brain slices. In addition, we surprisingly identified several cytosolic proteins with reg- ulatory functions, which are differentially expressed after treatment with $N$-propanoylmannosamine. Because sialic acid is the only monosaccharide that is activated in the nucleus, we hypothesize that transcription could be modulated by the unnatural CMP-Npropanoylneuraminic acid and that sialic acid activation might be a general tool to regulate cellular functions, such as neurite outgrowth.

Key words: N-propanoylmannosamine; neurite outgrowth; regeneration; sialylation; 2D-gel electrophoresis; MALDI-TOF MS
Sialic acids represent a family of amino sugars, which are components of complex $\mathrm{N}$ - and $\mathrm{O}$-glycans of glycoproteins and glycolipids. Sialylation of glycoproteins and glycolipids plays an important role during development, regeneration, and pathogenesis (Varki, 1993, 1997). Within the nervous system at times of extensive neuronal plasticity, e.g., during development or regeneration, the sialylation of glycoproteins and glycolipids differs from that found during tissue maintenance. One well characterized example is the unique polysialylation of the neural cell adhesion molecule (Finne et al., 1983; Sadoul et al., 1983; Santoni et al., 1988).

The biosynthesis of sialic acids starts in the cytosol. The physiological precursor of all sialic acids is $N$-acetylmannosamine (ManNAc). In previous studies we have shown that the novel nonphysiological $N$-propanoylmannosamine (ManNProp) is metabolized (like the physiological ManNAc) to $N$-propanoylneuraminic acid (Neu5Prop) in vitro and in vivo using the same metabolic route as ManNAc (see Scheme 1). The simple addition of ManNProp to the cell culture medium leads to the expression of Neu5Prop on cell surface glycoconjugates (Kayser et al., 1992; Keppler et al., 1995; Schmidt et al., 1998, 2000). This biochemical engineering, applied to different cell systems, has so far revealed several important biological functions of the $N$-acyl side chain of

\footnotetext{
Received May 20, 2002; revised July 1, 2002; accepted July 31, 2002.

This work was supported by the Deutsche Forschungsgemeinschaft (Ho 1959/3-1), the Schering Forschungsgesellschaft (B.B.), the Sonnenfeld-Stiftung, and the Fonds der Chemischen Industrie. We thank Ilona Danßmann for technical assistance.

*B.B. and C.K. contributed equally to this work.

Correspondence should be addressed to Dr. Rüdiger Horstkorte, Institut für Molekularbiologie und Biochemie, Fachbereich Humanmedizin, Freie Universität Berlin, Arnimallee 22, D-14195 Berlin-Dahlem, Germany. E-mail: rhorstko@ zedat.fu-berlin.de.

Copyright (C) 2002 Society for Neuroscience $\quad 0270-6474 / 02 / 228869-07 \$ 15.00 / 0$
}

sialic acid. Treatment of lymphoma cells with ManNProp reduced their infectibility by several sialic acid-dependent viruses, e.g., influenza A virus (Keppler et al., 1995). Human diploid lung fibroblasts displayed a loss of density-dependent growth control after biochemical engineering (Wieser et al., 1996). Treatment of neural cell cultures of newborn rats with ManNProp led to proliferation of astrocytes and microglia and increased the number of oligodendrocyte progenitor cells (Schmidt et al., 1998). These oligodendrocytes show calcium spiking in response to GABA after biochemical engineering of their cell surface with ManNProp (Schmidt et al., 2000). Biochemical engineering has not only been used to stimulate cells. This new method has been modified by the group of Carolyn Bertozzi. They used $N$-levulinoylmannosamine in which the acyl group contains a reactive ketone structure (Mahal et al., 1997). This enables the selective detection of the engineered cells and makes cells accessible for chemical modification. Furthermore they reported that $N$-butanoylmannosamine (ManNBut), a sialic acid precursor with an unnatural butanoyl residue, interferes with polysialylation of the neural cell adhesion molecule (Mahal et al., 2001).

One more important feature of engineered sialylation is an increase in the biological stability of glycoconjugates. The halflife of CEACAM-1, a member of the immunoglobulin superfamily, was increased after incorporation of Neu5Prop (Horstkorte et al., 2001; for review, see Keppler et al. 2001).

Here we report that the incorporation of $N$-propanoylneuraminic acid into cell surface glycoproteins of neuronal cell cultures results in a stimulation of neurite outgrowth. Furthermore, reestablishment of functional connections, such as the perforant pathway, is increased after incorporation of $N$-propanoylneuraminic acid. On the molecular level, we identified several regulatory proteins in the cytosol that were expressed differentially after biochemical engineering using ManNProp. 


\section{MATERIALS AND METHODS}

Cell culture. PC12-cells were routinely cultivated in Falcon plastic flasks using RPMI 1640 supplemented with 10\% horse serum. Differentiation (e.g., neurite outgrowth) was induced with a suboptimal concentration $(10 \mathrm{ng} / \mathrm{ml})$ of nerve growth factor (NGF) (Roche Biochemicals).

Small cerebellar granule cells were prepared as described (Keilhauer et al., 1985). In brief, coverslips were coated overnight with $0.01 \%$ poly-L-lysine or laminin at $37^{\circ} \mathrm{C}$ and washed three times with $\mathrm{H}_{2} \mathrm{O}$. Purified small $\left(3 \times 10^{5}\right)$ cerebellar neurons from 6- to 7-d-old mice were seeded onto each coverslip, yielding a final volume of $400 \mu$ l. Cultures were maintained for $20 \mathrm{hr}$.

Slices of entorhinal cortex and dentate gyrus were prepared from 6-d-old BALBC mice of either sex. Slices $(425 \mu \mathrm{m})$ were orientated as in vivo and fixed on microelectrode arrays (MEAs) (NMI, Reutlingen, Germany) of 60 substrate-integrated electrodes by a plasma clot. Cultures were maintained at a temperature of $36^{\circ} \mathrm{C}$ in $50 \%$ BMEM, $25 \%$ HBSS, and $25 \%$ horse serum containing $36 \mathrm{~mm}$ D-glucose and $1 \mathrm{~mm}$ L-glutamine. Medium was changed twice a week (Egert et al., 1998).

Analytical procedures. Protein was determined in 96-well ELISA plates using $200 \mu \mathrm{l}$ of bicinchonic acid protein reagent (Pierce, Rockford, IL) and a $50 \mu \mathrm{l}$ sample. Plates were evaluated in a 96-well ELISA reader (Spectra) at $570 \mathrm{~nm}$.

Preparation of cell extracts. Cell pellets were solubilized at $4^{\circ} \mathrm{C}$ for $1 \mathrm{hr}$ in buffer containing $150 \mathrm{~mm} \mathrm{NaCl}, 50 \mathrm{~mm}$ Tris, $1 \mathrm{mM} \mathrm{CaCl}_{2}, 1 \mathrm{~mm}$ $\mathrm{MgCl}_{2}, 1 \%$ Triton, and protease inhibitor mixture (Sigma, Deisenhofen, Germany) at $\mathrm{pH}$ 7.4. Solubilisates were centrifuged at $13000 \mathrm{rpm}$ for 30 min, and supernatants were collected.

Cells were homogenized in homogenization buffer by passing them 10 times through a syringe with a $22 \times 1.25$ ga needle. Homogenates were then centrifuged for $1 \mathrm{hr}$ at $100,000 \times g$, and the supernatants representing the cytosols were collected.

Quantification of N-acetyl acid and N-propanoylneuraminic acid. PC12cells were maintained for 1 or $3 \mathrm{~d}$ in the presence or absence of $5 \mathrm{~mm}$ ManNProp and then harvested and pelleted. Cell pellets $\left(10^{7}\right.$ cells) were lysed by hypotonic shock in distilled water and repeated freezing and thawing (two times). The crude membrane fractions were pelleted by centrifugation at $30,000 \times g$ for $20 \mathrm{~min}\left(4^{\circ} \mathrm{C}\right)$, and the pellets were lyophilized.

Quantification of total sialic acids. The pellet was washed twice with water and lyophilized. The content of membrane glycoconjugate-bound sialic acid was determined by hydrolyzing the pellet for $1 \mathrm{hr}$ with $2 \mathrm{M}$ acetic acid at $80^{\circ} \mathrm{C}$. Sialic acids were quantified by the thiobarbituric acid method (Aminoff, 1961) and HPLC analysis, as described (Keppler et al., 1995). Similar results were obtained by both methods.

Quantification of protein-bound N-acetyl-and N-propanoylneuraminic acid. Glycolipids were extracted using three different methanol/chloroform mixtures $(1: 2,1: 1,2: 1, \mathrm{v} / \mathrm{v})$ for $30 \mathrm{~min}$ each, followed by centrifugation at $10,000 \times g\left(30 \mathrm{~min}, 4^{\circ} \mathrm{C}\right)$. Glycoprotein-containing pellets were hydrolyzed, and sialic acids were purified and fluorescence labeled as described (Hara et al., 1987). Labeled sialic acids were chromatographed using a reversed phase C18 column (Lichrosorb C18, $5 \mu \mathrm{m}, 250 \times 4.6$ $\mathrm{mm}$; Knauer, Berlin, Germany) with a fluorescence detector (Ginkotek; excitation wavelength, $377 \mathrm{~nm}$; emission wavelength, $448 \mathrm{~nm}$ ). Eluent A contained distilled water, and eluent B contained acetonitrile/methanol $(60: 40, \mathrm{v} / \mathrm{v})$. The flow rate was $1 \mathrm{ml} / \mathrm{min}$. Separations were performed using a gradient running for the first $20 \mathrm{~min}$ in the isocratic mode with $10 \%$ eluent B. Eluent B was then raised to $25 \%$ within 25 min and finally to $50 \%$ within the subsequent $15 \mathrm{~min}$. Eluted neuraminic acids were identified by matrix-assisted laser desorption/ionization time-of-flight mass spectrometry (MALDI-TOF MS) and quantified using defined standards as already described (Keppler et al., 1995).

Quantification of neurite outgrowth. Cultures of PC12 cells or small cerebellar neurons were fixed and stained with cresyl violet. Photographs of each culture were taken randomly. Neurite outgrowth was quantified by computer-assisted process analysis (ITC, Kriftel, Germany) of at least 1500 cells per experiment (PC12 cells) or with the help of IP-Lab (NIH) software (small cerebellar neurons). Data were analyzed for significance by ANOVA.

Multi-electrode array. Beginning from $2 \mathrm{~d}$ in vitro (DIV), responses in cortex and dentate gyrus to electrical stimulation of layer II neurons of the entorhinal cortex were monitored at 2, 3, 4, 7, and 8 DIV. Electrophysiological activity was recorded simultaneously in 59 electrodes at 25 $\mathrm{kHz}$, stored, and analyzed off-line (hardware and software from Multichannel Systems). Responses of dentate gyrus neurons to electrical stimulation indicated reestablishment of the perforant pathway. The day of recovery was noted. Accumulated data for each substance were compared with control experiments in which no substance had been added to the culture medium (Hofmann et al., 2000) (analysis by NMI, Tübingen, Germany; see Figure $4 A$ for detail).

Two-dimensional-gel electrophoresis. Two-dimensional (2D)-gel electrophoresis was performed using the procedure as described previously (Löster and Kannicht, 2002). Cell extracts were mixed with 1.2-fold dry strip rehydration buffer to reach a final concentration of $2 \mathrm{M}$ thiourea, 7 M urea, 4\% (w/v) 3-[(3-cholamidopropyl)dimethylammonio]-1-propanesulfonate, $0.3 \%(\mathrm{w} / \mathrm{v})$ DTT, and $2 \%(\mathrm{v} / \mathrm{v})$ immobilized $\mathrm{pH}$ gradient (IPG) buffer, $\mathrm{pH} 4-7$. After a $30 \mathrm{~min}$ incubation at $25^{\circ} \mathrm{C}$ and a subsequent centrif ugation for $5 \mathrm{~min}$ at $12000 \mathrm{rpm}, \mathrm{pH} 4-7$ IPG strips $(18 \mathrm{~cm})$ (Amersham Biosciences, Freiburg, Germany) were rehydrated overnight at room temperature in $360 \mu \mathrm{l}$ volume of rehydration buffer/cell extract mixture. IEF was performed for $38,500 V_{\mathrm{h}}$ at a maximum of $3500 \mathrm{~V}$ using the Multiphor II system (Amersham Biosciences). After end of focusing, IPG strips were treated with equilibration buffer $[50 \mathrm{~mm}$ Tris, $6 \mathrm{~m}$ urea, $30 \%(\mathrm{v} / \mathrm{v})$ glycerol, $2 \%(\mathrm{w} / \mathrm{v}) \mathrm{SDS}$ ] supplemented with $0.15 \%(\mathrm{w} / \mathrm{v})$ DTT, followed by a secondary $15 \mathrm{~min}$ treatment with equilibration buffer supplemented with $0.24 \%(\mathrm{w} / \mathrm{v})$ iodoacetamide. The pretreated IPG strips were then transferred onto 5-15\% SDS-PAGE gels $(25 \times 20 \mathrm{~cm}$, $1.5 \mathrm{~mm}$, linear acrylamide gradient), and electrophoresis was performed overnight at a constant voltage of $100 \mathrm{~V}$ at $10^{\circ} \mathrm{C}$ according to the Amersham Biosciences instructions.

In situ digestion with trypsin and MALDI mass spectrometry. After 2D-electrophoresis, proteins were stained by colloidal Coomassie brilliant blue (Pierce). The spots of interest were cut off the gel, cut into small pieces, destained with $50 \%(\mathrm{v} / \mathrm{v})$ ethanol in aqua (aq.) bidest, washed extensively with aq. bidest to remove ethanol, and dried in a vacuum centrifuge. Trypsin (Trypsin, Sequencing Grade, Sigma) containing buffer (trypsin dissolved at $5 \mu \mathrm{g} / \mathrm{ml}$ in $100 \mathrm{~mm}$ Tris-HCI, $\mathrm{pH} 8.5$ ) was added to gel pieces. Protein digestion was performed overnight at $37^{\circ} \mathrm{C}$. Digestion was stopped by addition of $2.5 \%$ trifluoroacetic acid (TFA).

Supernatant and gel pieces were separated by centrifugation. Peptides were extracted and purified from supernatant by absorption onto a stationary reversed-phase matrix in pipette tips (ZipTipC18, Millipore, Eschborn, Germany) according to the instructions of the manufacturer. After five washes with $0.1 \%$ TFA in aq. bidest (v/v), bound peptides were eluted with $10 \mu$ l saturated matrix solution ( $\alpha$-cyano-4-hydroxycinnamic acid, Sigma) in $0.1 \%$ TFA (v/v) in $50 \%(\mathrm{v} / \mathrm{v})$ acetonitrile/water. One microliter of each eluted sample was applied to the target and allowed to dry at room temperature. MALDI-TOF MS was performed on a Bruker Biflex instrument (Bruker, Bremen, Germany). Ionization was accomplished with a $337 \mathrm{~nm}$ beam from a nitrogen laser. Mass spectra were recorded in the positive ion mode using the reflector. The masses of peptides were determined using adrenocorticotropic hormone fragment 18-39 (Sigma) and angiotensin II (Sigma) as internal standards.

\section{RESULTS}

Biochemical engineering of the acyl side chain of sialic acid, using ManNProp as an unnatural precursor, has been shown to stimulate glia cells and interfere with transmitter functions (Schmidt et al., 1998, 2000). In the present study, we inquired whether the most important prerequisite for regeneration of neural cells, namely neurite outgrowth, is affected by this new kind of biochemical engineering.

\section{Incorporation of Neu5Prop into the plasma membrane}

We first investigated whether neural cells are able to metabolize ManNProp and incorporate it as Neu5Prop on their cell surface (Scheme 1). For this purpose, PC12 cells were cultured for 1 or $3 \mathrm{~d}$ in the presence of $5 \mathrm{~mm}$ ManN Prop. To test whether PC12 cells synthesize Neu5Prop from the appropriate precursor (ManNProp), all sialic acids of membrane glycoproteins of ManNProp-treated PC12 cells were isolated and quantified by HPLC. When maintained for $1 \mathrm{~d}$ in the presence of ManNProp, $24 \%$ of the protein-bound sialic acids consisted of $N$-propanoylneuraninic acid, reaching $35 \%$ after 3 d (Fig. 1). 

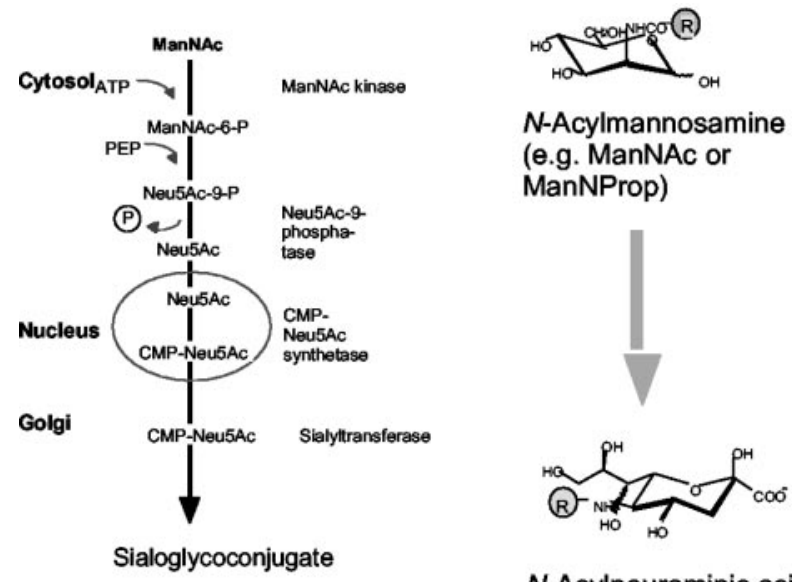

(e.g. ManNAc or

ManNProp)

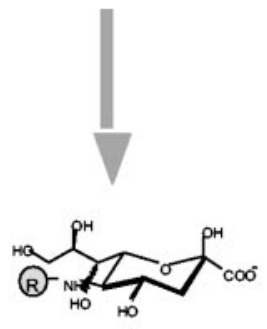

N-Acylneuraminic acid

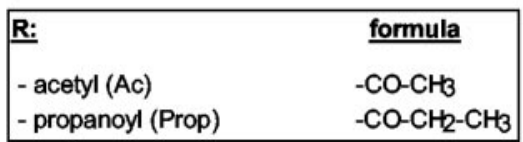

Scheme 1. Biosynthetic pathway of physiological and engineered sialic acid precursors.

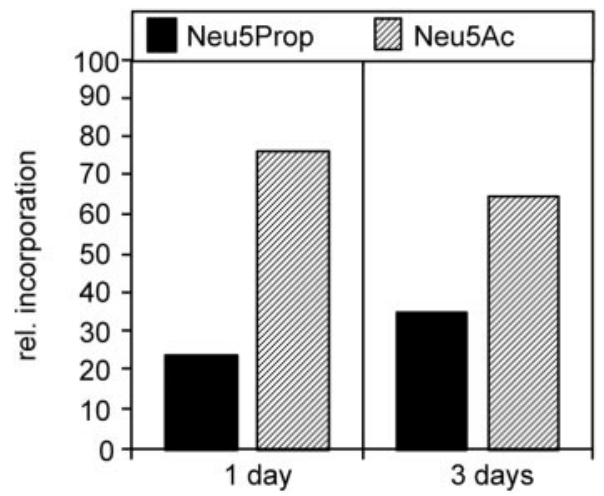

Figure 1. Incorporation of $N$-propanoylneuraminic acid in PC12 cells. PC12 cells were incubated in the presence of ManNProp for different time periods. Incorporated $N$-propanoylneuraminc acid (Neu5Prop) was quantified by HPLC analysis and compared with physiological sialic acid (Neu5Ac).

\section{Biochemical engineering does not increase cell surface sialylation}

To determine whether the treatment of ManNAc or ManNProp leads to increased overall sialylation, we quantified the total cell surface-bound sialic acids of PC12 cells cultured in the absence and presence of ManNAc or ManNProp. PC12 cells were cultivated for $48 \mathrm{hr}$ in the absence or presence of $5 \mathrm{~mm}$ ManNAC or ManNProp, respectively. We found that treatment of PC12 cells with ManNAc led to a slightly increased sialylation (Table 1); treatment with ManNProp resulted in a nonsignificant increase of cell surface sialylation (Table 1).

\section{Biochemical engineering stimulates neurite outgrowth of PC12 cells}

Rat PC12 cells have been widely used as a standard system to study neurite outgrowth. These cells express neural cell adhesion molecule in its nonpolysialylated form (Horstkorte et al., 1999)
Table 1. Quantification of total sialic acids in PC12 cells cultured in the absence or presence of ManNac or ManNProp

\begin{tabular}{lr} 
Culture condition & \multicolumn{1}{c}{$\mathrm{nmol} / \mathrm{mg}$} \\
\hline No additive & $8.8 \pm 1.8$ \\
ManNAc & $11.8 \pm 0.3$ \\
ManNProp & $9.0 \pm 0.5$
\end{tabular}

Each value is represented by four experiments.

and respond to NGF by extending neurites via a Ras-dependent pathway. We first quantified neurite outgrowth of PC12 cells, grown in the absence or presence of ManNProp, on different substrates.

PC12 cells were cultured in the presence of suboptimal concentrations of NGF on poly-D-lysine, collagen I, or laminin. The best neurite outgrowth was observed on laminin. In the presence of $0.5 \mathrm{~mm}$ ManNProp, PC12 cells had nearly $30 \%$ longer neurites on laminin compared with control cultures without ManNProp. Neurite outgrowth was not stimulated on collagen I and poly-Dlysine. At an increased concentration of ManNProp of $5 \mathrm{~mm}$, neurite outgrowth was stimulated on laminin by $69 \%$ and to a lesser extent also on collagen (14\%), but not on poly-D-lysine. In the presence of $25 \mathrm{~mm}$ ManNProp, neurite outgrowth was stimulated on laminin (61\%), collagen (21\%), and poly-D-lysine $(18 \%)$. In another set of experiments, PC12 cells were grown in the presence of the $5 \mathrm{~mm}$ ManNAc (the physiological precursor of sialic acid) (Fig. 2B). ManNAc is also capable of stimulating neurite outgrowth on laminin, but not on collagen or poly-Dlysine and to a much lesser extent compared with ManNProp (Fig. 2B). Figure $2 C$ shows two representative micrographs of PC12 cells grown in the absence or presence of $25 \mathrm{~mm}$ ManNProp.

As demonstrated in Figure 2, the maximal response of PC12 cells was at $5 \mathrm{~mm}$ ManNProp. We therefore performed subsequent experiments in the presence of $5 \mathrm{~mm}$ ManNProp.

\section{Biochemical engineering stimulates neurite outgrowth of small cerebellar granule cells}

Using collagen I or laminin as substrate, we analyzed neurite outgrowth of small cerebellar granule cells in vitro in the absence or presence of ManNProp or ManNAc (Fig. 3).

Analysis of $>600$ cells showed that neurite outgrowth of small cerebellar granule cells was also stimulated by ManNProp (Fig. $3 A)$. When cultures were grown in the presence of $5 \mathrm{~mm}$ ManN Prop on collagen I, neurite outgrowth was stimulated by $25 \%$ compared with control cultures. However, when small cerebellar granule cells were grown on laminin in the presence of ManNProp, neurite outgrowth was stimulated by $>120 \%$ (Fig. 3A). Again, the physiological precursor of sialic acid (ManNAc) also stimulated neurite outgrowth, but this stimulation was only half of that observed after biochemical engineering with ManN Prop (Fig. 3A).

\section{Reestablishment of the perforant pathway}

Establishment of functional connections is a basic requirement for the correct interaction of neurons of the CNS. To test the regenerative capacity of ManNProp, i.e., to stimulate the reestablishment of connections within central nervous tissue, organotypic cocultures were combined with extracellular multi-electrode recording technology (Fig. 4A,B). Four preparations, each with 15 cocultures of entorhinal cortex and dentate gyrus, were started. Figure $4 C$ illustrates the percentage of explants showing recovery 


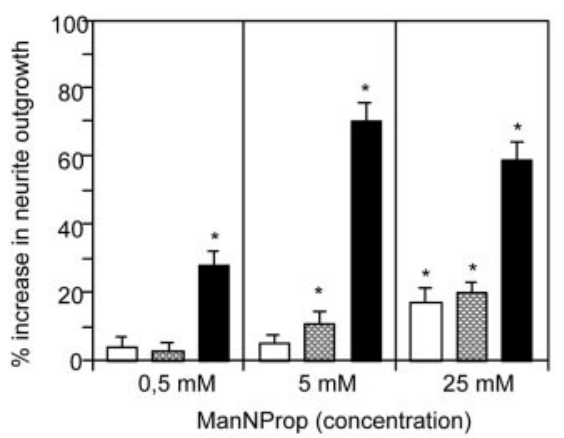

B

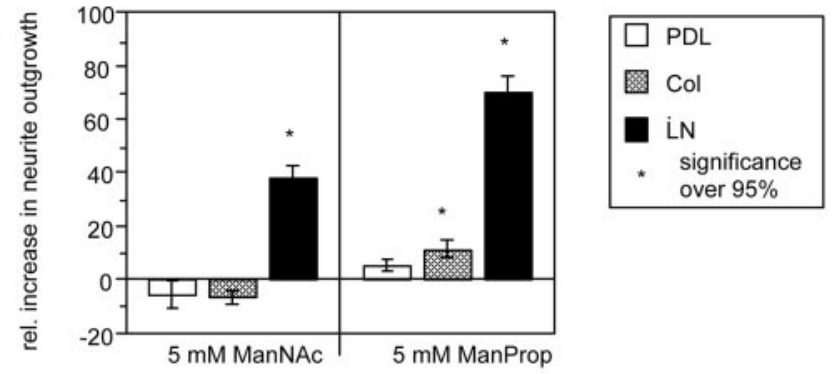

C
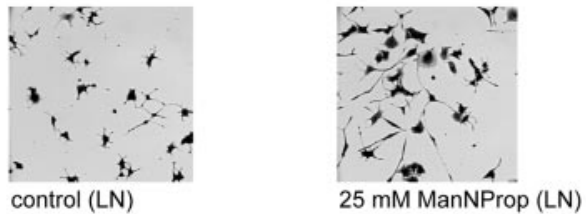

Figure 2. Stimulation of neurite outgrowth in PC12 cells. $A, \mathrm{PC} 12$ cells were grown in the presence of $0.5,5$, or $25 \mathrm{~mm}$ ManNProp on poly-Dlysine $(P D L)$, collagen I $(\mathrm{Col})$, or laminin $(L N)$. Neurite outgrowth was quantified and is expressed as percentage increase over control. Error bars represent mean values \pm SD of 25 micrographs containing at least 25 cells each. $B, \mathrm{PC} 12$ cells were grown in the presence of $5 \mathrm{~mm}$ ManN Prop or ManNAc on poly-D-lysine $(P D L)$, collagen I $(\mathrm{Col})$, or laminin $(L N)$. Neurite outgrowth was quantified and expressed as percentage increase over control. Error bars represent mean values \pm SD of 25 micrographs containing at least 25 cells each. $C$, Representative micrographs of PC12 cultures cultured on laminin $(L N)$ grown in the absence (control ) or presence of $25 \mathrm{~mm}$ nonphysiological $N$-propanoylmannosamine (ManNProp).

of the perforant pathway on each culture day. After $2 \mathrm{~d}$ of culture, $7 \%$ of the explants showed recovery of the perforant pathway. However, when the explants were cultured in the presence of ManNProp, 36\% of the explants showed recovery after $2 \mathrm{~d}$ in culture. In control experiments in the presence of ManNAc, 25\% of the explants showed recovery (Fig. 4C); 100\% recovery was attained after $7 \mathrm{~d}$ in the presence of ManNProp and after $8 \mathrm{~d}$ in control and ManNAc cultures (data not shown).

\section{Proteome analysis of engineered PC12 cells}

Which molecular mechanisms underlie the stimulation of neurite outgrowth? Neurite outgrowth is a complex mechanism involving the intracellular signal transduction machinery and the expression of novel genes. Therefore, we stimulated PC12 cells with 5 mm ManNProp and compared the expression patterns of cytosolic proteins. Cytosolic fractions of PC12 cells grown in the absence or presence of ManNProp were prepared and subjected to

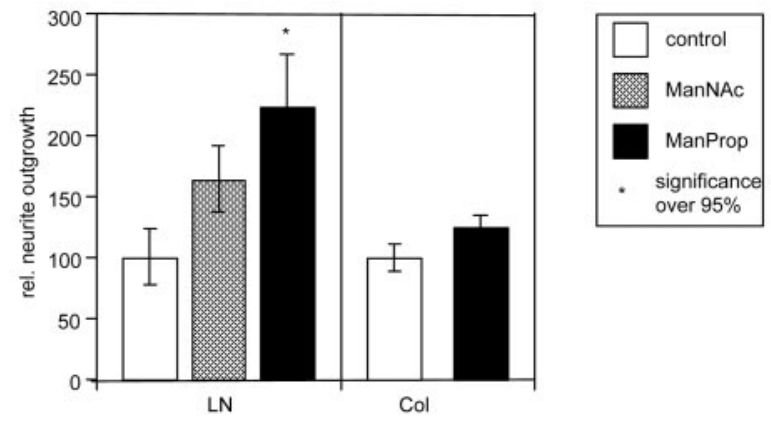

B

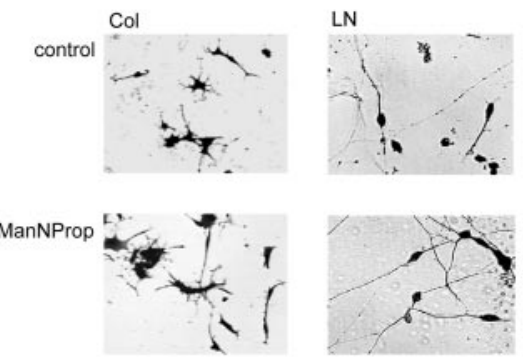

Figure 3. Stimulation of neurite outgrowth of small cerebellar granule cells. $A$, Small cerebellar granule cells were grown in the absence or presence of $5 \mathrm{~mm}$ ManN Prop or ManNAc on collagen I $(\mathrm{Col})$ or laminin $(L N)$. Neurite length was quantified and set at $100 \%$ in the absence of additives. Error bars represent mean values \pm SD of 20 micrographs containing at least 15 cells each. $B$, Representative micrographs of small cerebellar granule cells cultured on collagen I $(\mathrm{Col})$ or laminin $(L N)$ grown in the absence (control) or presence of $5 \mathrm{~mm}$ ManNProp.

2-D gel electrophoresis. Gels were stained with Coomassie blue and analyzed. Figure 5 shows a representative 2-D gel of 15 gels that were used for these analyses. We compared the proteins from gels of PC12 cells grown in the absence or presence of ManNProp and selected 12 proteins with significantly altered expression. Corresponding spots were cut out of the gels and in-gel digested with trypsin. Tryptic peptides were eluted from the gel and further analyzed by MALDI-TOF MS. Ten proteins could be identified; from the other two no matching peptides were found in the swissprot-databank. Figure 5 shows the position of these proteins on the 2D-gels, and Table 2 summarizes all data of the spot analysis. The identified proteins form two major groups: the first group represents proteins involved in the regulation of growth and development, such as ULIP protein, 14-3-3 proteins, and heat shock proteins. Interestingly, with the exception of the heat shock protein 27 , the expression of all proteins is downregulated by treatment of the PC12 cells with $5 \mathrm{~mm}$ ManNProp. The second group consists of enzymes such as $\alpha$ enolase, tyrosine-3monooxygenase, and ubiquitin $\mathrm{C}$-terminal hydrolase isoenzyme L1. These enzymes are involved in general cellular functions and regulation of proteolysis.

\section{DISCUSSION}

This study demonstrates that the $N$-acyl side chain of sialic acid is a potent tool for stimulating neuronal cells. After incorporation of the unnatural $N$-propanoylneuraminic acid into cell surface glycoconjugates, both PC12 cells and small cerebellar granule cells showed increased neurite outgrowth in vitro. In addition, regeneration, as shown by the reestablishment of the perforant 
A

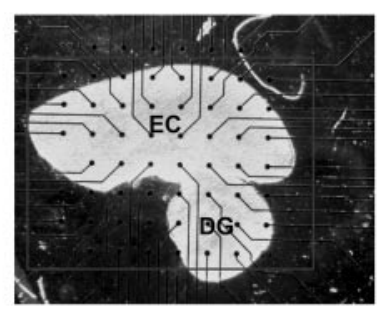

B

C

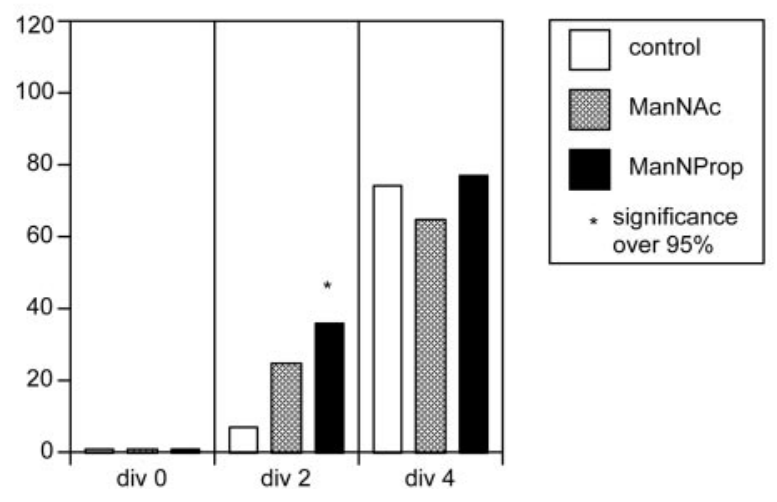

Figure 4. Reestablishment of the perforant pathway. $A$, Coculture of entorhinal cortex $(E C)$ and dentate gyrus $(D G)$ after $2 \mathrm{~d}$ in culture on microelectrode array. Electrodes have a spacing of $200 \mu \mathrm{m}$ and a diameter of $30 \mu \mathrm{m}$ each. Not all electrodes are covered by tissue. $B$, Electrophysiological activity recorded in both slices simultaneously. Preparation is the same as in $A$. The electrode marked by the asterisk was used as the stimulating electrode. $C$, Cocultures of entorhinal cortex and dentate gyrus were grown in the absence (control) and presence of ManNAC or ManN Prop. Bars represent percentage of cultures that are reestablished after days in culture (div) as indicated.

pathway in slice cultures, was also stimulated. The increased neurite outgrowth was accompanied by a changed protein expression pattern.

Our experiments show that neurite outgrowth and regeneration are stimulated by the unnatural sialic acid precursor, ManNProp, but also to a lesser degree by the physiological sialic acid precursor, ManNAc. These data correspond to earlier observations that not only ManNProp but to a lesser extent also ManNAc stimulated the enrichment of A2B5-positive oligodendrocytes, and both were also stimulators of astrocyte proliferation (Schmidt et al., 1998). This might be explained by a constitutive undersialylation of the investigated cells in culture, because application of both precursors, ManNAc or ManNProp, respectively, led to a slightly increased cell surface sialylation (Table 1) (Keppler et al., 1999, Mantey et al., 2001). It remains to be elucidated whether increased sialylation is beneficial per se to regeneration in vivo. The stimulation of neurite outgrowth was twice as high when cells were biochemically engineered with the unnatural ManNProp compared with cells treated with the physiological precursor of sialic acid. This increased neurite outgrowth is the specific effect of the prolonged $N$-acyl side chain of sialic acid, e.g., biochemical engineering.

This stimulation of neurite outgrowth by ManNProp is matrix dependent. It is much better on laminin than on collagen I or poly-D-lysine, which suggests an involvement of integrin recep- tors. It has been shown in various experiments that $\beta 1$-integrins are regulators for neurite outgrowth (Treubert and Brummendorf, 1998; Ivins et al., 2000; Werner et al., 2000). Biochemical engineering of the side chain of sialic acid might activate $\beta 1$ integrins. It has been shown that $\beta 1$-integrins can be activated by removal of sialic acid; treatment with sialidases increases the adhesion of HL60 cells to fibronectin (Pretzlaff et al., 2000). This activation might be one explanation for the specific stimulation of neurite outgrowth on laminin induced by ManNProp treatment. The differences between laminin and collagen substrates might be the result of different $\alpha$ integrins. PC12 cells express mainly $\alpha 1$ $\beta 1$, and $\alpha 3 \beta 1$ integrins, which are receptors for both laminin and collagen (Tomaselli et al., 1990). In contrast, cerebellar neurons express $\alpha 1 \quad \beta 1$ as a collagen/laminin receptor and $\alpha 6 \beta 1$ as receptor for laminin (Hall et al., 1997).

In most of our experiments, we used 5 mm ManNProp, because this concentration supported maximal stimulation. Such a high concentration is necessary because membranes are not permeable for ManNProp, and no transport mechanism exists. Preliminary data suggest that peracetylation of ManNProp enables it to cross membranes and could help to reduce the necessary concentration of ManNProp by a factor of 100 . Nevertheless, even a high concentration of ManN Prop does not affect the viability of any of the cells investigated so far (Keppler et al., 2001).

NGF-mediated neurite outgrowth in PC12 cells is controlled via Ras and the MAP-kinase pathway (Szeberenyi et al., 1990; Fukuda et al., 1995). Therefore, we decided to investigate the expression of cytosolic proteins in PC12 cells before and after stimulation with ManNProp. This strategy was intended to throw light on the molecular intracellular mechanism underlying the ManNProp-stimulated neurite outgrowth. In previous studies we have already shown that treatment of cerebellar explants with ManNProp leads to an increased expression of the A2B5 epitope (Schmidt et al., 1998).

Some of the molecules, identified in PC12 cells after stimulation of neurite outgrowth with ManNProp, are involved in neurite outgrowth. The role of 14-3-3 proteins as potential regulators of neurite outgrowth has been debated for many years (for review, see Fu et al., 2000). They are associated with GABA receptors (Couve et al., 2001), which are known to be modulated by ManNProp, leading to calcium spiking in oligodendrocytes (Schmidt et al., 2000). Furthermore, 14-3-3 proteins are associated with sialyltransferase IV (Gao et al., 1996), suggesting that 14-3-3 proteins might be involved in both neurite outgrowth and biosynthesis of sialoglycoconjugates.

Unc-33-like phosphoprotein (ULIP) is involved in axon guidance and outgrowth (Quinn et al., 1999). Interestingly, we also identified ULIP as a target of ManNProp treatment. Although the general expression of ULIP correlates with neurite outgrowth, we measured a downregulation of ULIP expression in response to ManNProp.

This is the first evidence that biochemical engineering of the acyl side chain of sialic acid not only influences cell surface receptors via expression of protein-bound unnatural sialic acids, but that ManNProp also influences the expression of cytosolic proteins that are involved in signal transduction. The mechanism whereby ManNProp changes protein expression will be the object of future investigations. In contrast to all other monosaccharides, which are activated in the cytosol, sialic acid is activated to CMP-sialic acid in the nucleus (Coates et al., 1980; Kean, 1991). Therefore it might be possible that transcription could be modulated by the unnatural CMP-Neu5Prop in the nucleus. 


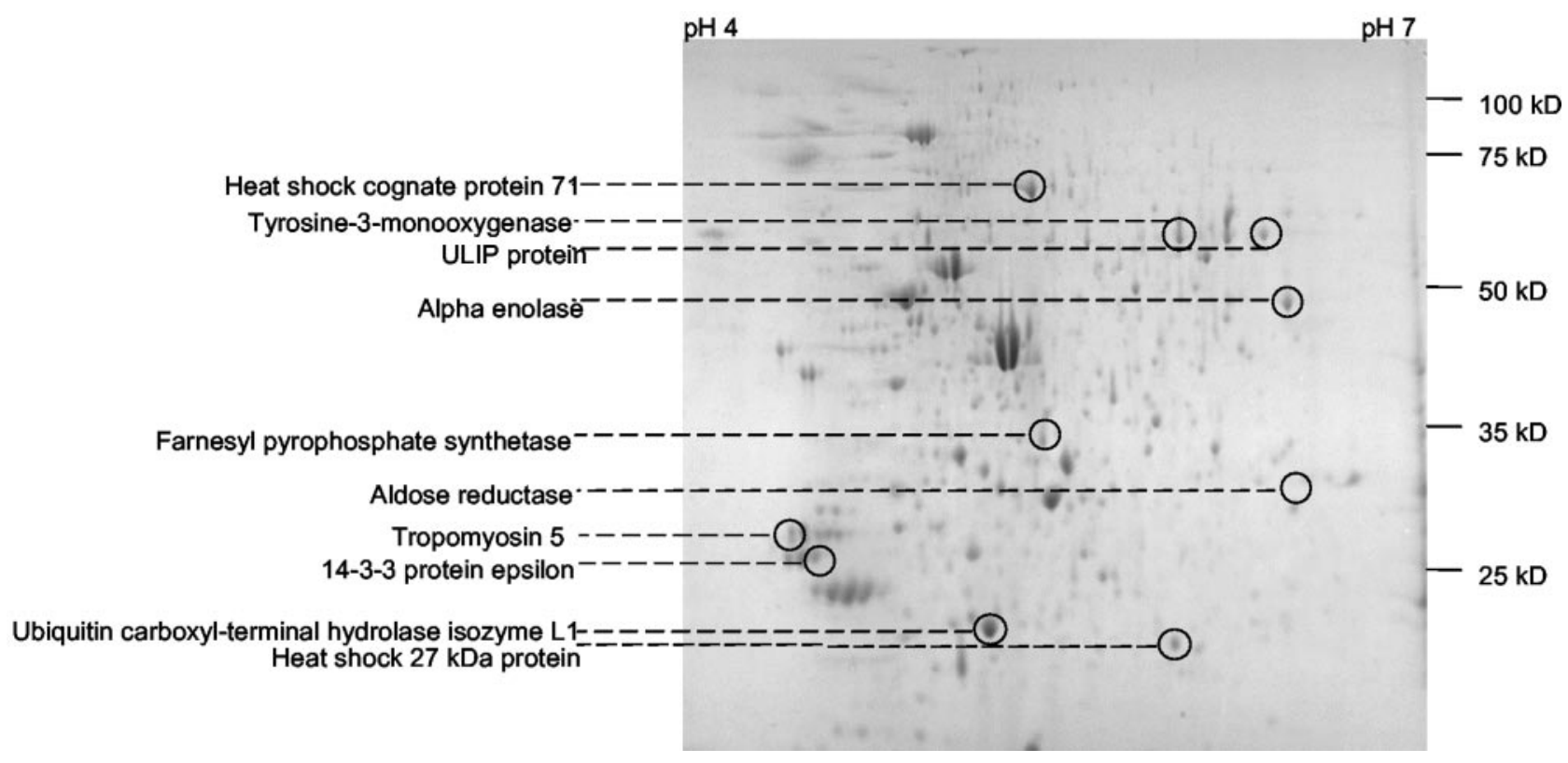

Figure 5. 2D-Gel electrophoresis of cytosolic proteins of PC12 cells.

Table 2. Proteins identified by MALDI-TOF MS

\begin{tabular}{|c|c|c|c|c|c|c|c|c|}
\hline Protein & Species & MW & $\begin{array}{l}\text { Theoretical } \\
\text { MW }\end{array}$ & PI & $\begin{array}{l}\text { Theoretical } \\
\text { PI }\end{array}$ & $\begin{array}{l}\text { Number of } \\
\text { matched } \\
\text { peptides }\end{array}$ & $\begin{array}{l}\% \text { sequence } \\
\text { coverage }\end{array}$ & $\begin{array}{l}\text { Upregulation/ } \\
\text { downregulation }\end{array}$ \\
\hline$\alpha$ enolase & Rat & 48000 & 49984 & 6.5 & 6.16 & 13 & 35.8 & Downregulation \\
\hline ULIP protein & Mouse & 60000 & 61936 & 6.3 & 6.04 & 9 & 20.4 & Downregulation \\
\hline Tyrosine-3-monooxygenase & Rat & 59000 & 55966 & 5.8 & 5.74 & 18 & 51.6 & Downregulation \\
\hline $\begin{array}{l}\text { Heat shock cognate pro- } \\
\text { tein } 71\end{array}$ & Rat & 70000 & 70804 & 5.4 & 5.24 & 15 & 31.7 & Downregulation \\
\hline Farnesyl pyrophosphate & & & & & & & & \\
\hline synthetase & Rat & 35000 & 40829 & 5.5 & 5.9 & 16 & 39.1 & Downregulation \\
\hline 14-3-3 protein $\epsilon$ & Rat & 27000 & 29173 & 4.5 & 4.63 & 13 & 53.7 & Downregulation \\
\hline $\begin{array}{l}\text { Ubiquitin C-terminal hy- } \\
\text { drolase isozyme L1 }\end{array}$ & Rat & 23000 & 24782 & 5.4 & 5.12 & 8 & 45.7 & Downregulation \\
\hline Tropomyosin 5 & Mouse & 27000 & 29020 & 4.8 & 4.75 & 16 & 59.7 & Downregulation \\
\hline Heat shock $27 \mathrm{kDa}$ protein & Rat & 22000 & 22892 & 6.1 & 6.12 & 13 & 56.8 & Upregulation \\
\hline Aldose reductase & Rat & 34000 & 35666 & 6.5 & 6.28 & 10 & 49.5 & Downregulation \\
\hline
\end{tabular}

MW, Molecular weight; PI, isoelectric point.

On the basis of all these results, we propose a novel mechanism for the stimulation of neurite outgrowth via biochemical engineering of the acyl side chain of sialic acid.

\section{REFERENCES}

Aminoff D (1961) A rapid and sensitive method for the quantitation of microgram quantities of protein utilizing the principle of protein-dye binding. Anal Biochem 72:248-254.

Coates SW, Gurney Jr T, Sommers LW, Yeh M, Hirschberg CB (1980) Subcellular localization of sugar nucleotide synthetases. J Biol Chem 255:9225-9229.

Couve A, Kittler JT, Uren JM, Calver AR, Pangalos MN, Walsh FS, Moss SJ (2001) Association of GABA(B) receptors and members of the 14-3-3 family of signaling proteins. Mol Cell Neurosci 17:317-328.

Egert U, Schlosshauer B, Fennrich S, Nisch W, Fejtl M, Knott T, Muller T, Hämmerle H (1998) A novel organotypic long-term culture of the rat hippocampus on substrate-integrated multielectrode arrays. Brain Res Brain Res Protoc 2:229-242.

Finne J, Finne U, Deagostini-Bazin H, Goridis C (1983) Occurrence of alpha 2-8 linked polysialosyl units in a neural cell adhesion molecule. Biochem Biophys Res Commun 112:482-487.
Fu H, Subramanian RR, Masters SC (2000) 14-3-3 proteins: structure, function, and regulation. Annu Rev Pharmacol Toxicol 40:617-647.

Fukuda M, Gotoh Y, Tachibana T, Dell K, Hattori S, Yoneda Y, Nishida E (1995) Induction of neurite outgrowth by MAP kinase in PC12 cells. Oncogene 11:239-244.

Gao L, Gu XB, Yu DS, Yu RK, Zeng G (1996) Association of a 14-3-3 protein with CMP-NeuAc:GM1 alpha 2,3-sialyltransferase. Biochem Biophys Res Commun 224:103-107.

Hall H, Carbonetto S, Schachner M (1997) L1/HNK-1 carbohydrateand beta 1 integrin-dependent neural cell adhesion to laminin-1. J Neurochem 68:544-553.

Hara S, Takemori Y, Yamaguchi M, Nakamura M, Ohkura Y (1987) Fluorometric high-performance liquid chromatography of $N$-acetyland $N$-glycolylneuraminic acids and its application to their microdetermination in human and animal sera, glycoproteins, and glycolipids. Anal Biochem 164:138-145.

Hofmann F, Leibrock C, Volkmer H, Hämmerle H (2000) Functional establishment of the perforant pathway in an organotypic co-culture monitored over two weeks with a microelectrode array. Res Neurol Neurosci 16:54.

Horstkorte R, Le $\beta$ ner N, Gerardy-Schahn R, Lucka L, Danker K, Reutter W (1999) Expression of the polysialyltransferase, ST8SiaIV: 
polysialylation interferes with adhesion and differentiation in vitro. Exp Cell Res 246:122-128.

Horstkorte R, Lee HY, Lucka L, Danker K, Mantey L, Reutter W (2001) Biochemical engineering of the side chain of sialic acids increases the biological stability of the highly sialylated cell adhesion molecule CEACAM1. Biochem Biophys Res Commun 283:31-35.

Ivins JK, Yurchenco PD, Lander AD (2000) Regulation of neurite outgrowth by integrin activation. J Neurosci 20:6551-6560.

Kayser H, Geilen CC, Paul C, Zeitler R, Reutter W (1992) Incorporation of $N$-acyl-2-amino-2-deoxy-hexoses into glycosphingolipids of the pheochromocytoma cell line PC 12. J Biol Chem 267:16934-16938.

Kean EL (1991) Sialic acid activation. Glycobiology 1:441-447.

Keilhauer G, Faissner A, Schachner M (1985) Differential inhibition of neurone-neurone, neurone-astrocyte and astrocyte-astrocyte adhesion by L1, L2 and N-CAM antibodies. Nature 316:728-730.

Keppler OT, Stehling P, Herrmann M, Kayser H, Grunow D, Reutter W, Pawlita M (1995) Biosynthetic modulation of sialic acid-dependent virus-receptor interactions of two primate polyoma viruses. J Biol Chem 270:1308-1314.

Keppler OT, Hinderlich S, Langner J, Schwartz-Albiez R, Reutter W, Pawlita M (1999) UDP-GlcNAc 2-epimerase: a regulator of cell surface sialylation. Science 284:1372-1376.

Keppler OT, Horstkorte R, Pawlita M, Schmidt C, Reutter W (2001) Biochemical engineering of the $N$-acyl side chain of sialic acid: biological implications. Glycobiology 11:11R-18R.

Löster K, Kannicht C (2002) 2D-Electrophoresis: detection of glycosylation and influence on spot pattern. In: Posttranslational modifications of proteins-tools for functional proteomics (Kannicht C, ed), pp 301-316. Totowa, NJ: Humana.

Mahal LK, Yarema KJ, Bertozzi CR (1997) Engineering chemical reactivity on cell surfaces through oligosaccharide biosynthesis. Science 276:1125-1128.

Mahal LK, Charter NW, Angata K, Fukuda M, Koshland Jr DE, Bertozzi CR (2001) A small-molecule modulator of poly-alpha 2,8-sialic acid expression on cultured neurons and tumor cells. Science 294:380-381.

Mantey LR, Keppler OT, Pawlita M, Reutter W, Hinderlich S (2001) Efficient biochemical engineering of cellular sialic acids using an unphysiological sialic acid precursor in cells lacking UDP- $N$ acetylglucosamine 2-epimerase. FEBS Lett 503:80-84.

Pretzlaff RK, Xue VW, Rowin ME (2000) Sialidase treatment exposes the beta1-integrin active ligand binding site on HL60 cells and increases binding to fibronectin. Cell Adhes Commun 7:491-500.

Quinn CC, Gray GE, Hockfield S (1999) A family of proteins implicated in axon guidance and outgrowth. J Neurobiol 41:158-164.

Sadoul R, Hirn M, Deagostini-Bazin H, Rougon G, Goridis C (1983) Adult and embryonic mouse neural cell adhesion molecules have different binding properties. Nature 304:347-349.

Santoni MJ, Goridis C, Fontecilla-Camps JC (1988) Molecular modeling of the immunoglobulin-like domains of the neural cell adhesion molecule (NCAM): implications for the positioning of functionally important sugar side chains. J Neurosci Res 20:304-310.

Schmidt C, Stehling P, Schnitzer J, Reutter W, Horstkorte R (1998) Biochemical engineering of neural cell surfaces by the synthetic $N$-propanoyl-substituted neuraminic acid precursor. J Biol Chem 273:19146-19152.

Schmidt C, Ohlemeyer C, Kettenmann H, Reutter W, Horstkorte R (2000) Incorporation of $N$-propanoylneuraminic acid leads to calcium oscillations in oligodendrocytes upon the application of GABA. FEBS Lett 478:276-280.

Szeberenyi J, Cai H, Cooper GM (1990) Effect of a dominant inhibitory Ha-ras mutation on neuronal differentiation of PC12 cells. Mol Cell Biol 10:5324-5332.

Tomaselli KJ, Hall DE, Flier LA, Gehlsen KR, Turner DC, Carbonetto S, Reichardt LF (1990) A neuronal cell line (PC12) expresses two beta 1 -class integrins-alpha 1 beta 1 and alpha 3 beta 1 -that recognize different neurite outgrowth-promoting domains in laminin. Neuron $5: 651-662$.

Treubert U, Brummendorf T (1998) Functional cooperation of $\beta 1$ integrins and members of the Ig superfamily in neurite outgrowth induction. J Neurosci 18:1795-805.

Varki A (1993) Biological roles of oligosaccharides: all of the theories are correct. Glycobiology 3:97-130.

Varki A (1997) Sialic acids as ligands in recognition phenomena. FASEB J 11:248-255.

Werner A, Willem M, Jones LL, Kreutzberg GW, Mayer U, Raivich G (2000) Impaired axonal regeneration in $\alpha 7$ integrin-deficient mice. J Neurosci 20:1822-1830.

Wieser JR, Heisner A, Stehling P, Oesch F, Reutter W (1996) In vivo modulated $N$-acyl side chain of $N$-acetylneuraminic acid modulates the cell contact-dependent inhibition of growth. FEBS Lett 395:170-173. 